TRANSACTIONS OF THE

AMERICAN MATHEMATICAL SOCIETY

Volume 185, November 1973

\title{
MULTIPLICATIVE STRUCTURE OF GENERALIZED KOSZUL COMPLEXES
}

BY

EUGENE H. GOVER

\begin{abstract}
A multiplicative structure is defined for the generalized Koszul complexes $K\left(\wedge^{p} f\right)$ associated with the exterior powers of a map $f: R^{m} \rightarrow R^{n}$ where $R$ is a commutative ring and $m \geq n$. With this structure $K\left(\wedge^{n} f\right)$ becomes a differential graded $R$-algebra over which each $K\left(\wedge^{p} f\right), 1 \leq p \leq n$, is a DG right $K\left(\wedge^{n} f\right)$-module. For $f=0$ and $n>1$, the multiplication and all higher order Massey operations of $K\left(\wedge^{n} f\right)$ are shown to be trivial. When $R$ is noetherian local, $K\left(\wedge^{n} f\right)$ is used to define a class of local rings which includes the local complete intersections. The rings obtained for $n>1$ are CohenMacaulay but not Gorenstein. Their Betti numbers and Poincaré series are computed but these do not characterize the rings.
\end{abstract}

Introduction. Unlike the Koszul complex which comes equipped with the underlying structure of an exterior algebra, the generalized Koszul complexes defined by Buchsbaum in [2] and Buchsbaum-Rim in [5] have lacked an additional multiplicative structure. It is the main purpose of this paper to provide that structure for certain generalized Koszul complexes and by so doing to make them into differential graded algebras such that the remaining complexes become differential graded (right) modules over these algebras. The enriched structure makes possible a simple proof of a previously known result concerning the annihilators of the homologies of generalized Koszul complexes. In addition, it suggests generalizations of various concepts which have made use of the exterior algebra structure of the usual Koszul complex. Thus we are led to consider those local rings which generalize the notion of a local complete intersection and to study several homological invariants of such rings. These rings are CohenMacaulay but since they are not in general Gorenstein, they do not provide a class of rings intermediate between local complete intersections and Gorenstein rings. However, they do have readily computable Betti numbers and rational Poincaré series, and so provide another example of a class of local rings for which this information has been obtained.

In $\$ 1$ we fix some of the notation used in subsequent sections and review basic properties of generalized Koszul complexes.

$\$ 2$ presents a related complex originally defined by Eagon and Northcott in [7]. A chain map is constructed from that complex to a suitable generalized Koszul

Received by the editors August 10, 1972.

AMS (MOS) subject classifications (1970). Primary 13D99, 13H10, 15A78; Secondary 16A62.

Key words and phrases. Generalized Koszul complex, differential graded algebra, trivial multiplicative structure, Massey operations, generalized local complete intersection, Betti numbers, Poincaré series.

Copyright $\odot 1974$, American Mathematical Society 
complex which induces an isomorphism on the homology level. The nature of the chain map shows that all cycles in the generalized Koszul complex are homologous to symmetric cycles in a certain minimal subcomplex.

$\$ 3$ contains the main results of the paper in its Theorems 3.6 and 3.9. The first establishes the existence of a multiplicative structure for generalized Koszul complexes; the second states that for those generalized Koszul complexes defined by a zero map and which are differential graded algebras, the multiplication as well as all higher order Massey operations are trivial.

In $\$ 4$ these results are exploited in the case of local rings. Certain factor rings of regular local rings called generalized local complete intersections are defined and studied in terms of several homological invariants. It is shown that such rings are Golod rings in the sense of [8] and so have explicitly computed Betti numbers and Poincare series. Finally, an example is given to show that none of the invariants discussed is by itself sufficient to characterize these generalized complete intersections.

Throughout this paper, all rings will be commutative with an identity element and all modules will be unitary. Module homomorphisms will usually be referred to as maps.

The author wishes to express his gratitude to David Buchsbaum for his many helpful suggestions and for providing valuable assistance as supervisor of the author's doctoral dissertation, upon which much of the material in the present paper is based.

1. Generalized Koszul complexes. We begin by reviewing some of the basic facts about generalized Koszul complexes. Further details can be found in [2], [5] and [3].

Let $R$ be a commutative ring with identity. Given an $R$-module $A$, denote by $A^{*}$ its dual and by $\wedge A$ and $\wedge A^{*}$ the corresponding exterior algebras over $R$. For each pair of nonnegative integers $p$ and $q$ define maps $\omega_{A}: \wedge^{q} A^{*} \otimes \wedge^{p} A$ $\rightarrow \wedge^{p-q} A$ and $\omega_{A^{*}}^{\prime}: \wedge^{q} A \otimes \wedge^{p} A^{*} \rightarrow \wedge^{p-q} A^{*}$ as follows: if $q=1, \alpha \in A^{*}$, and $a=a_{1} \wedge \cdots \wedge a_{p} \in \wedge^{p} A$, let

$$
\omega_{A}(\alpha \otimes a)=\sum_{i=1}^{p}(-1)^{i+1} \alpha\left(a_{i}\right) \cdot a_{1} \wedge \cdots \wedge \hat{a}_{i} \wedge \cdots \wedge a_{p} .
$$

We will let $\alpha(a)$ stand for $\omega_{A}(\alpha \otimes a)$. Observe that if $\alpha_{1}, \alpha_{2} \in A^{*}$, then $\alpha_{1} \alpha_{2}(a)$ $=-\alpha_{2} \alpha_{1}(a)$ which permits us to define $\omega_{A}\left(\alpha_{1} \wedge \cdots \wedge \alpha_{q} \otimes a\right)=\alpha_{1} \cdots \alpha_{q}(a)$ for decomposable elements $\alpha=\alpha_{1} \wedge \cdots \wedge \alpha_{q} \in \wedge^{q} A^{*}$. We then extend the definition of $\omega_{A}$ by linearity to all of $\wedge^{q} A^{*} \otimes \wedge^{p} A$. Again, we write the image of the map as $\alpha(a)$. Define $\omega_{A^{\prime}}^{\prime}$ to be the composite

$$
\wedge^{q} A \otimes \wedge^{p} A^{*} \rightarrow \wedge^{q} A^{* *} \otimes \wedge^{p} A^{*} \stackrel{\omega^{\infty}}{\longrightarrow} \wedge^{p-q} A^{*}
$$

where the first map is induced by the natural map from $A$ to $A^{* *}$ on the first factor and is the identity map on the second factor. 
Given an $R$-module homomorphism $f: A \rightarrow B$ let $f^{*}, \wedge f$, and $\wedge f^{*}$ denote the induced maps on the appropriate duals and exterior algebras. Define $\omega_{f \bullet}$ : $\wedge^{q} B^{*} \otimes \wedge^{P} A \rightarrow \wedge^{p-q} A$ by $\beta \otimes a \mapsto \omega_{A}\left(\wedge f^{*}(\beta) \otimes a\right)$ and $\omega_{f}: \wedge^{q} A \otimes \wedge^{p} B^{*}$ $\rightarrow \wedge^{p-q} B^{*}$ by $a \otimes \beta \mapsto \omega_{B^{*}}^{\prime}(\wedge f(a) \otimes \beta)$. Once again we abbreviate the images of these maps by $\beta(a)$ and $a(\beta)$ respectively.

Consider now the following slight modification of a reduced Bar complex. Let the complex be $\mathcal{B}(\wedge f)$ with

$$
\mathcal{B}_{0}(\wedge f)=\wedge A, \quad \mathcal{B}_{t}(\wedge f)=\left(\otimes_{\imath} \overline{\wedge B^{*}}\right) \otimes \wedge A \text { for } t>0
$$

where $\otimes_{t}$ stands for the $t$-fold tensor product, $\overline{\wedge B^{*}}=\Sigma_{s>0} \wedge^{s} B^{*}$, and all tensor products are taken over $R$. The boundary maps $d_{l}^{\prime}: \mathcal{B}_{t}(\wedge f) \rightarrow$ $\mathcal{B}_{t-1}(\wedge f)$ are defined by setting

$$
\begin{array}{r}
d_{t}^{\prime}\left(\beta_{1} \otimes \cdots \otimes \beta_{t} \otimes a\right)=\sum_{i=1}^{t-1}(-1)^{i+1} \beta_{1} \otimes \cdots \otimes \beta_{i} \wedge \beta_{i+1} \\
\otimes \cdots \otimes \beta_{t} \otimes a \\
+(-1)^{t+1} \beta_{1} \otimes \cdots \otimes \beta_{t-1} \otimes \beta_{t}(a)
\end{array}
$$

Alternatively, these maps may be defined by the recursive formulas

$$
\begin{aligned}
d_{1}^{\prime}(\beta \otimes a) & =\beta(a), \\
d_{1}^{\prime}\left(\beta_{1} \otimes \beta_{2} \otimes \gamma\right) & =\beta_{1} \wedge \beta_{2} \otimes \gamma-\beta_{1} \otimes d_{t-1}^{\prime}\left(\beta_{2} \otimes \gamma\right),
\end{aligned}
$$

where $\gamma \in \mathcal{B}_{t-2}(\wedge f)$. It is then easy to show that $\left(d^{\prime}\right)^{2}=0$.

We next define subcomplexes $\mathcal{B}^{q}\left(\wedge^{p} f\right)$ of $\mathcal{B}(\wedge f)$ for each pair of positive integers $p$, q. Let $\mathbb{B}_{f}^{q}\left(\wedge^{p} f\right)=\wedge^{p} A \subset \mathcal{B}_{0}(\wedge f)$. Then build a subcomplex of $\mathcal{B}(\wedge f)$ for a given $q$ by defining

$$
\mathcal{B}_{i}^{q}\left(\wedge^{p} f\right)=\sum_{s_{1} \geq q ; s_{1}>0} \wedge^{s_{1}} B^{*} \otimes \cdots \otimes \wedge^{s_{1}} B^{*} \otimes \wedge^{p+\Sigma s_{1}} A, \quad t>0,
$$

and $d_{t}^{q, p}=d_{t}^{\prime}$ suitably restricted. Suppose that $B$ is such that $\wedge^{n+1} B=\wedge^{n+1} B^{*}$ $=0$. Then for $q=n+1-p$ it is possible to lengthen the complex $B^{n+1-p}\left(\wedge^{p} f\right)$ by adding the term $\wedge^{P} f: \wedge^{P} A \rightarrow \wedge^{P} B$ at the end. This can be done since $\left(\wedge^{p} f\right) \circ d_{1}^{n+1-p, p}=0$ by virtue of the fact that $\wedge^{n+1} f=0$.

We can now define the generalized Koszul complexes of Buchsbaum [2] and Buchsbaum-Rim [5].

Definition. Consider a map $f: A \rightarrow B$ where $B$ is such that $\wedge^{n+1} B=\wedge^{n+1} B^{*}$ $=0$ for some positive $n$. The generalized Koszul complex associated with the map $\wedge^{P} f, 1 \leq p \leq n$, denoted $K\left(\wedge^{P} f\right)$, is defined by setting

$$
\begin{aligned}
K_{0}\left(\wedge^{p} f\right) & =\wedge^{p} B, & d_{1} & =\wedge^{p} f \\
K_{t}\left(\wedge^{p} f\right) & =\mathcal{B}_{t-1}^{n+1-p}\left(\wedge^{p} f\right), & d_{t+1} & =d_{t}^{n+1-p_{1} p} \text { for } t>0 .
\end{aligned}
$$


Thus $K\left(\wedge^{p} f\right)$ has the form

$$
\begin{aligned}
\cdots \rightarrow & \sum_{s_{1} \geq n+1-p ; s_{2}>0} \wedge^{s_{1}} B^{*} \otimes \wedge^{s_{2}} B^{*} \otimes \wedge^{p+\Sigma s_{1} A} \\
& \stackrel{d_{3}}{\longrightarrow} \sum_{s \geq n+1-p} \wedge^{s} B^{*} \otimes \wedge^{p+s} A \\
& \stackrel{d_{s}}{\longrightarrow} \wedge^{p} A \stackrel{\Lambda_{f}^{\prime}}{\longrightarrow} \wedge^{p} B .
\end{aligned}
$$

If $A$ and $B$ are free $R$-modules of rank $m$ and $n$ respectively, $m \geq n$, then the complexes $K\left(\wedge^{p} f\right), 1 \leq p \leq n$, each have length $m-n+1$. The following theorem from [2], [5], and [6] lists three basic properties of these generalized Koszul complexes.

Theorem 1.1. Consider a map $f: R^{m} \rightarrow R^{n}, m \geq n$, and let

$$
I(f)=\text { Ann Coker } \wedge^{n} f .
$$

Then, for each $p$ with $1 \leq p \leq n$,

(a) $I(f) \subset$ Ann $H\left(K\left(\wedge^{p} f\right)\right)$.

\section{If $R$ is noetherian,}

(b) $m-n+1-$ depth $I(f)=$ the largest integer $q$ for which $H_{q}\left(K\left(\wedge^{p} f\right)\right)$

$\neq 0$. In particular, $K\left(\wedge^{p} f\right)$ is acyclic if and only if depth $I(f)=m-n+1$.

(c) $K\left(\wedge^{p} f\right)$ is rigid, i.e., if $H_{i}\left(K\left(\wedge^{p} f\right)\right)=0$ then $H_{j}\left(K\left(\wedge^{p} f\right)\right)=0$ for all $j>i$.

2. A minimal subcomplex of $K\left(\wedge^{n} f\right)$. Let $f$ be as in the hypotheses of 1.1. An alternate grade sensitive complex of length $m-n+1$ associated with the map $\wedge^{n} f: \wedge^{n} R^{m} \rightarrow \wedge^{n} R^{n}$ was defined by Eagon and Northcott in [7]. In this section we define that complex and construct an explicit chain map from it to $K\left(\wedge^{n} f\right)$ which induces an isomorphism on the homology level. Grade sensitivity in the generic case already implies that the two complexes have isomorphic homologies. The construction of a specific chain map inducing the isomorphism shows in addition that all cycles in $K\left(\wedge^{n} f\right)$ are homologous to symmetric cycles of lowest possible total degree in a minimal subcomplex.

Given an arbitrary $R$-module homomorphism $f: A \rightarrow B$ and a pair of positive integers $p, q$ it is possible to construct a complex $c^{q}\left(\wedge^{p} f\right)$ by setting

$$
\begin{aligned}
& c_{i}^{q}\left(\wedge^{p} f\right)=S_{t}\left(B^{*}\right) \otimes \wedge^{p+(q-1)+t} A \text { for } t>0 \text { and } \\
& c_{q}^{q}\left(\wedge^{p} f\right)=\wedge^{p+q-1} A
\end{aligned}
$$

where $S\left(B^{*}\right)$ is the symmetric algebra of $B^{*}$. Buchsbaum and Eisenbud [4] have shown how to obtain the boundary maps for $C^{q}\left(\wedge^{p} f\right)$ in a basis-free manner by constructing this complex as the dual of a certain subcomplex of the bigraded algebra $\wedge A^{*} \otimes S(B)$ in which the map $f$, identified with an element of $A^{*} \otimes B$, becomes a bihomogeneous element of bidegree 1,1 such that $f^{2}=0$. However, 
to facilitate the construction of a chain map from $C^{q}\left(\wedge^{p} f\right)$ to $B^{q}\left(\wedge^{p} f\right)$, we will assume $B^{*}$ is free of rank $n$ and has the basis $\xi_{1}, \ldots, \xi_{n}$. Any element in $S_{t}\left(B^{*}\right)$ can then be written as a homogeneous form of degree $t$ in $\xi_{1}, \ldots, \xi_{n}$. For such a form $F_{t}$, let $F_{t}\left(\hat{\xi}_{i}\right)$ be that part of $F_{t}$ which is expressible as a form in $\xi_{1}, \ldots, \hat{\xi}_{i}, \ldots, \xi_{n}$. Then $F_{t}-F_{t}\left(\hat{\xi}_{i}\right)$ is divisible by $\xi_{i}$ and so we can define $D_{i} F_{t} \in S_{t-1}\left(B^{*}\right)$ to be the form $\left(F_{t}-F_{t}\left(\hat{\xi}_{i}\right)\right) / \xi_{i}$. Clearly $D_{i} D_{j} F_{t}=D_{j} D_{i} F_{t}$. The boundary maps $\partial_{t}^{q, p}: C_{t}^{q}\left(\wedge^{p} f\right) \rightarrow C_{i-1}^{q}\left(\wedge^{p} f\right)$ can now be defined by setting

$$
\partial_{i}^{q, p}\left(F_{t} \otimes a\right)=\sum_{i=1}^{n} D_{i} F_{t} \otimes \xi_{i}(a) \text { for } t>i
$$

and

$$
\partial_{1}^{q, p}\left(F_{1} \otimes a\right)=\sum_{i=1}^{n} D_{i} F_{1} \cdot \xi_{i}(a)
$$

A straightforward calculation shows that $\left(\partial^{q, p}\right)^{2}=0$.

As in the preceding section, the fact that $\wedge^{n+1} B^{*}=0$ means that the complex $C^{n+1-p}\left(\wedge^{p} f\right)$ can be augmented by the term $\wedge^{n} f: \wedge^{n} A \rightarrow \wedge^{n} B$. We note that in the present situation, $C^{n+1-p}\left(\wedge^{p} f\right)=C^{l}\left(\wedge^{n} f\right)$ for each $p, 1 \leq p \leq n$.

Definition. Given $f: A \rightarrow B$ with $B$ a free $R$-module of rank $n$, the complex $C\left(\wedge^{n} f\right)$ is defined by setting

$$
\begin{aligned}
& C_{0}\left(\wedge^{n} f\right)=\wedge^{n} B, \quad \partial_{1}=\wedge^{n} f, \\
& C_{t}\left(\wedge^{n} f\right)=C_{t-1}^{n+1-p}\left(\wedge^{p} f\right), \quad \partial_{t+1}=\partial_{t}^{n+1-p, p} \text { for } t>0 .
\end{aligned}
$$

If $A$ is free of rank $m, m \geq n$, then $C\left(\wedge^{n} f\right)$ is the complex of length $m-n+1$ originally defined by Eagon and Northcott [7]. Theorem 1.1 holds for $C\left(\wedge^{n} f\right)$. When the ring $R$ is local with maximal ideal $\mathrm{m}, C\left(\wedge^{n} f\right)$ has the further property of being minimal in the sense that $\partial\left(C\left(\wedge^{n} f\right)\right) \subset \mathfrak{m} \cdot C\left(\wedge^{n} f\right)$.

We now suppose that $f: R^{m} \rightarrow R^{n}, m \geq n$, is a map, and observe that both $K\left(\wedge^{n} f\right)$ and $C\left(\wedge^{n} f\right)$ can be obtained from corresponding generic complexes in the following way. Form the polynomial ring $S=R\left[X_{11}, \ldots, X_{m n}\right]$ and let $g(m, n): S^{m} \rightarrow S^{n}$ be the map whose matrix is $\left(X_{i j}\right)$. It then follows that $K\left(\wedge^{p} f\right)=K\left(\wedge^{p} g(m, n)\right) \otimes_{S} \bar{S}$ and $C\left(\wedge^{n} f\right)=C\left(\wedge^{n} g(m, n)\right) \otimes_{S} \bar{S}$ where $\bar{S}$ $=S /\left(X_{i j}-a_{i j}\right)$ and $\left(a_{i j}\right)$ is a matrix representation for $f$. A key observation of [6] was that

$$
H_{*}\left(K\left(\wedge^{p} f\right)\right)=\operatorname{Tor}^{S}\left(\text { Coker } \wedge^{p} g(m, n), \bar{S}\right)
$$

the result following from Northcott's proof that $I(m, n)=$ Ann Coker $\wedge^{n} g(m, n)$ is perfect and $\operatorname{depth}(I(m, n) ; S)=m-n+1$. Since it is equally true that $H_{*}\left(C\left(\wedge^{n} f\right)\right)=\operatorname{Tor}^{S}\left(\right.$ Coker $\left.\wedge^{n} g(m, n), \bar{S}\right)$, we have established 
Proposition 2.1. $H_{*}\left(K\left(\wedge^{n} f\right)\right) \approx H_{*}\left(C\left(\wedge^{n} f\right)\right)$.

We next construct a specific chain map $\varphi: C\left(\wedge^{n} f\right) \rightarrow K\left(\wedge^{n} f\right)$ which induces this isomorphism. Toward that end we define the map

$$
\varphi_{l}^{q}: C_{l}^{l}\left(\wedge^{n+q} f\right) \rightarrow\left(\otimes_{l} R^{n *}\right) \otimes \wedge^{n+q+\imath} R^{m} \subset \mathcal{B}_{l}^{l}\left(\wedge^{n+q} f\right)
$$

for each integer $q, 0 \leq q \leq m-n$, by the following recursive formulas:

$$
\begin{aligned}
\varphi_{0}^{q} \text { and } \varphi_{1}^{q} & =\text { the appropriate identity maps, } \\
\varphi_{t}^{q}\left(F_{t} \otimes a\right) & =(-1)^{t+1} \sum_{i=1}^{n} \xi_{i} \otimes \varphi_{t-1}^{q+1}\left(D_{i} F_{t} \otimes a\right) \\
& =-\sum_{i_{1}, i_{2}} \xi_{i_{1}} \otimes \xi_{i_{2}} \otimes \varphi_{t-2}^{q+2}\left(D_{i_{2}} D_{i_{1}} F_{t} \otimes a\right) \text { for } t>1 .
\end{aligned}
$$

$\left\{\varphi_{t}^{q}\right\}_{t \geq 0}$ defines a map $\varphi^{q}: C^{l}\left(\wedge^{n+q} f\right) \rightarrow \mathcal{B}^{1}\left(\wedge^{n+q} f\right)$.

Proposition 2.2. For each $q, 0 \leq q \leq m-n, \varphi^{q}$ is a chain map.

Proof. For each $t>0$ and for each $q, 0 \leq q \leq m-n$, we must show that $d_{t}^{q} \circ \varphi_{t}^{q}=\varphi_{t-1}^{q} \circ \partial_{l}^{q}$ where $d^{q}=d^{1, n+q}$ and $\partial^{q}=\partial^{1, n+q}$. The proof is by induction on $t$ and $q$.

Consider first $F_{1} \otimes a \in R^{n *} \otimes \wedge^{n+q+1} R^{m}=C_{l}\left(\wedge^{n+q} f\right)$.

$$
\begin{aligned}
d_{1}^{q} \circ \varphi_{1}^{q}\left(F_{1} \otimes a\right) & =d_{1}^{q}\left(F_{1} \otimes a\right)=F_{1}(a) \\
& =\sum_{i=1}^{n} D_{i} F_{1} \cdot \xi_{i}(a)=\varphi_{0}^{q} \circ \partial_{1}^{q}\left(F_{1} \otimes a\right) .
\end{aligned}
$$

Consequently $d_{1}^{q} \circ \varphi_{1}^{q}=\varphi^{q} \circ \partial_{1}^{q}$ which establishes the relationship for $t=1$ and for all $q$.

Now make the induction assumption that $d_{j}^{q} \circ \varphi_{j}^{q}=\varphi_{j-1}^{q} \circ \partial_{j}^{q}$ for all $j<t$ and for all q. Choose $F_{t} \otimes a \in S_{t}\left(R^{n^{*}}\right) \otimes \wedge^{n+q+\imath} R^{m}=C_{i}^{l}\left(\wedge^{n+q} f\right)$. We then have

$$
\begin{aligned}
d_{t}^{q} \circ \varphi_{i}^{q}\left(F_{t} \otimes a\right)= & -d_{i}^{q}\left(\sum_{i_{1}, i_{2}} \xi_{i_{1}} \otimes \xi_{i_{2}} \otimes \varphi_{i-2}^{q+2}\left(D_{i_{2}} D_{i_{1}} F_{t} \otimes a\right)\right) \\
= & -\sum_{i_{1}, i_{2}} \xi_{i_{1}} \wedge \xi_{i_{2}} \otimes \varphi_{i-2}^{q+2}\left(D_{i_{2}} D_{i_{1}} F_{t} \otimes a\right) \\
& +\sum_{i_{1}, i_{2}} \xi_{i_{1}} \otimes d_{i-1}^{q+1}\left(\xi_{i_{2}} \otimes \varphi_{t-2}^{q+2}\left(D_{i_{2}} D_{i_{1}} F_{t} \otimes a\right)\right) \\
= & \sum_{i_{1}, i_{2}} \xi_{i_{1}} \otimes d_{i-1}^{q+1}\left(\xi_{i_{2}} \otimes \varphi_{i-2}^{q+2}\left(D_{i_{2}} D_{i_{1}} F_{t} \otimes a\right)\right) \\
= & (-1)^{t} \sum_{i_{1}} \xi_{i_{1}} \otimes d_{t-1}^{q+1}\left(\varphi_{i-1}^{q+1}\left(D_{i_{1}} F_{t} \otimes a\right)\right) \\
= & (-1)^{t} \sum_{i_{1}} \xi_{i_{1}} \otimes \varphi_{i-2}^{q+1}\left(\partial_{t-1}^{q+1}\left(D_{i_{1}} F_{t} \otimes a\right)\right) \\
= & (-1)^{t} \sum_{i_{1}, i_{2}} \xi_{i_{1}} \otimes \varphi_{i-2}^{q+1}\left(D_{i_{2}} D_{i_{1}} F_{t} \otimes \xi_{i_{2}}(a)\right),
\end{aligned}
$$


where the fourth from the last equality holds because the sum is symmetric in $i_{1}$ and $i_{2}$ which implies that

$$
\sum_{i_{1}, i_{2}} \xi_{i_{1}} \wedge \xi_{i_{2}} \otimes \varphi_{i-2}^{q+2}\left(D_{i_{2}} D_{i_{1}} F_{t} \otimes a\right)=0 .
$$

The next to the last equality follows from the induction assumption. On the other hand,

$$
\begin{aligned}
\varphi_{i-1}^{q} \circ \partial_{i}^{q}\left(F_{i} \otimes a\right) & =\varphi_{i-1}^{q}\left(\sum_{i} D_{i} F_{i} \otimes \xi_{i}(a)\right) \\
& =(-1)^{t} \sum_{i_{1}, i_{2}} \xi_{i_{2}} \otimes \varphi_{i-2}^{q+1}\left(D_{i_{2}} D_{i_{1}} F_{t} \otimes \xi_{i_{1}}(a)\right) .
\end{aligned}
$$

Consequently, $d_{t}^{q} \circ \varphi_{t}^{q}=\varphi_{t-1}^{q} \circ \partial_{t}^{q}$ which establishes the relationship for $t$ and for all $q$.

Corollary 2.3. Define $\varphi: C\left(\wedge^{n} f\right) \rightarrow K\left(\wedge^{n} f\right)$ by setting $\varphi_{0}=1_{K_{0}\left(\wedge^{n} f\right) \text { and } \varphi_{t}}$ $=\varphi_{t-1}^{0}$ for $t>0$. Then $\varphi$ is a chain map and $\varphi_{*}$, the induced map on the homology level, is an isomorphism.

Proof. To show that $\varphi$ is a chain map, we need only check that $\varphi$ commutes with the boundaries in the lowest dimensions. But in that case, $\varphi_{0}$ and $\varphi_{1}$ are identity maps while $\partial_{1}=d_{1}=\wedge^{n} f$ so it is certainly true that $d_{1} \circ \varphi_{1}=\varphi_{0} \circ \partial_{1}$.

To see that $\varphi_{*}$ is an isomorphism, we observe that the corresponding chain map in the generic case, $\varphi(m, n)$, specializes to $\varphi$. That is,

$$
\varphi=\varphi(m, n) \otimes_{S} 1_{\bar{S}}: C\left(\wedge^{n} g(m, n)\right) \otimes_{S} \bar{S} \rightarrow K\left(\wedge^{n} g(m, n)\right) \otimes_{S} \bar{S}
$$

Since $\varphi(m, n)$ lifts the identity in dimension zero and both $C\left(\wedge^{n} g(m, n)\right)$ and $K\left(\wedge^{n} g(m, n)\right)$ are $S$-free resolutions of $I(m, n)$, it follows that $\varphi_{*}$ is an isomorphism.

Definition. Let $\bar{K}\left(\wedge^{n} f\right)$ be the subcomplex $\varphi\left(C\left(\wedge^{n} f\right)\right)$ of $K\left(\wedge^{n} f\right)$.

Remark 2.4. $\varphi$ is clearly a monomorphism which means that $\bar{K}\left(\Lambda^{n} f\right)$ is a minimal subcomplex of $K\left(\wedge^{n} f\right)$ and is isomorphic to $C\left(\wedge^{n} f\right)$. Since

$$
\varphi\left(C_{t}\left(\wedge^{n} f\right)\right) \subset\left(\otimes_{t-1} R^{n *}\right) \otimes \wedge^{n+t-1} R^{m}
$$

$\bar{K}_{t}\left(\wedge^{n} f\right)$ is generated by all symmetric sums of the form $\Sigma_{0} \beta_{\sigma(1)} \otimes \cdots \otimes \beta_{o(t-1)}$ $\otimes a$ where $\sigma$ is a permutation of $t-1$ elements and $\operatorname{deg} \beta_{\alpha(i)}=1$ for each $i$. Corresponding to the well-known fact that the symmetric tensor power of a free module is a direct summand of the tensor power, we therefore have that $\bar{K}_{t}\left(\wedge^{n} f\right)$ is a direct summand of $K_{t}\left(\wedge^{n} f\right)$ for each $t$.

Corollary 2.5. Any cycle in $K_{t}\left(\wedge^{n} f\right)$ is homologous to a cycle contained in $\bar{K}_{t}\left(\wedge^{n} f\right)$. 
Proof. Let $z$ be a cycle in $K_{t}\left(\wedge^{n} f\right)$. Since $\varphi_{*}$ is an isomorphism by 2.3,

$$
\left(\varphi_{t}\right)_{*} \circ\left(\varphi_{t}\right)_{*}^{-1}([z])=[z]
$$

where $[z]$ is the homology class of $z$. But $\operatorname{Im} \varphi_{t}=\bar{K}_{t}\left(\wedge^{n} f\right)$ from which it follows that $z$ must be homologous to a cycle in $\bar{K}_{t}\left(\wedge^{n} f\right)$.

3. A multiplicative structure for generalized Koszul complexes. In this section we construct a multiplication for $K\left(\wedge^{n} f\right)$ making that complex into a differential graded $R$-algebra. The complexes $K\left(\wedge^{p} f\right), 1 \leq p \leq n$, then turn out to be differential graded $K\left(\wedge^{n} f\right)$-modules. One consequence of the multiplicative structure is a simple proof of the fact that Ann Coker $\wedge^{n} f \subset$ Ann $H\left(K\left(\wedge^{p} f\right)\right.$ ). for each $p, 1 \leq p \leq n$ (Theorem 1.1, part (a)). Another is that when $f$ is the zero map, $K\left(\wedge^{n} f\right)$ has a trivial multiplication and trivial higher order Massey operations. We begin by setting up the needed machinery and in so doing, make use of several of the maps defined by Buchsbaum in [3].

For each $R$-module $A, \wedge A$ and $\wedge A^{*}$ are commutative, cocommutative Hopf algebras with coproducts

$$
\Delta: \wedge A \rightarrow \wedge A \otimes \wedge A
$$

and

$$
\Delta^{*}: \wedge A^{*} \rightarrow \wedge A^{*} \otimes \wedge A^{*}
$$

If $x \in \wedge A$ we will write $\Delta(x)=\sum_{i} x_{i} \otimes x_{i}^{\prime}$ where $\operatorname{deg} x_{i}^{\prime}=\operatorname{deg} x-\operatorname{deg} x_{i}$. Since $\Delta$ is associative, the $p$-fold iterated coproduct $\Delta^{p}: \wedge A \rightarrow \otimes_{p+1} \wedge A$ is a well-defined map. If now $\left(n_{1}, \ldots, n_{p+1}\right)$ is a $(p+1)$-tuple of nonnegative integers, define the map

$$
\Delta^{p}\left(n_{1}, \ldots, n_{p+1}\right): \wedge A \rightarrow \wedge^{n_{1}} A \otimes \cdots \otimes \wedge^{n+1} A
$$

to be the composite $\pi\left(n_{1}, \ldots, n_{p+1}\right) \circ \Delta^{p}$ where $\pi\left(n_{1}, \ldots, n_{p+1}\right)$ is the projection $\otimes_{p+1} \wedge A \rightarrow \wedge^{n_{1}} A \otimes \cdots \otimes \wedge^{n_{p+1}} A$. If $x \in \wedge^{s} A$, we will write $\Delta(t, s-t)(x)$ $=\sum_{i} x_{i, t} \otimes x_{i}^{\prime}$ where $\operatorname{deg} x_{i, t}=t$ and $\operatorname{deg} x_{i}^{\prime}=s-t$. It should be noted that the definition of the map $\Delta^{p}\left(n_{1}, \ldots, n_{p+1}\right)$ given here differs from the definition given in [3] where $\Delta^{p}\left(n_{1}, \ldots, n_{p+1}\right)$ is the map which in present notation is written

$$
\sum_{0 \leq s_{1}<n_{1}} \Delta^{p}\left(s_{1}, \ldots, s_{p+1}\right): \wedge A \rightarrow \sum_{0 \leq s_{1}<n_{1}} \wedge^{s_{1}} A \otimes \cdots \otimes \wedge^{s_{p+1}} A \text {. }
$$

Consider briefly the situation where $\Lambda_{i}$ and $\Gamma_{i}$ are commutative $R$-algebras and where for each $i, \omega_{i}: \Lambda_{i} \otimes \Gamma_{i} \rightarrow \Gamma_{i}$ is an $R$-module homomorphism. Let

$$
\overline{\omega_{1} \otimes \cdots \otimes \omega_{p}}: \Lambda_{1} \otimes \cdots \otimes \Lambda_{p} \otimes \Gamma_{1} \otimes \cdots \otimes \Gamma_{p} \rightarrow \Gamma_{1} \otimes \cdots \otimes \Gamma_{p}
$$


be the composite $\left(\omega_{1} \otimes \cdots \otimes \omega_{p}\right) \circ T_{1, \ldots, p}$ where the map

$$
T_{1, \ldots, p}: \Lambda_{1} \otimes \cdots \otimes \Lambda_{p} \otimes \Gamma_{1} \otimes \cdots \otimes \Gamma_{p} \rightarrow\left(\Lambda_{1} \otimes \Gamma_{1}\right) \otimes \cdots \otimes\left(\Lambda_{p} \otimes \Gamma_{p}\right)
$$

is obtained by repeated use of the transposition maps $T: \Lambda_{i} \otimes \Gamma_{j} \rightarrow \Gamma_{j} \otimes \Lambda_{i}$, in turn defined by $T(\alpha \otimes \beta)=(-1)^{\operatorname{deg} \alpha \cdot \operatorname{deg} \beta} \beta \otimes \alpha$.

We now once again introduce the map $f: A \rightarrow B$ where $A$ and $B$ are free $R$ modules of respective ranks $m$ and $n, m \geq n$.

Definition. For each $x \in \wedge A$ define $\bar{\omega}_{x}^{p}:\left(\otimes_{p} \wedge B^{*}\right) \otimes \wedge A \rightarrow\left(\otimes_{p} \wedge B^{*}\right)$ $\otimes \wedge A$ by

$$
\begin{aligned}
\bar{\omega}_{x}^{p}\left(\beta_{1} \otimes \cdots \otimes \beta_{p} \otimes y\right) \\
\quad=\overline{\left(\otimes_{p} \omega_{f}\right) \otimes \mu}\left[\sum_{i_{1}<t_{i} ;<\infty} \Delta^{p}\left(s_{1}, \ldots, s_{p}, s\right)(x) \otimes \beta_{1} \otimes \cdots \otimes \beta_{p} \otimes y\right]
\end{aligned}
$$

where $\beta_{i} \in \bigwedge^{t} B^{*}, y \in \wedge A, \mu$ is the exterior multiplication on $\wedge A$, and $\omega_{f}$ is as defined at the beginning of $\S 1$.

In a similar manner it is possible to define a map $\otimes_{p} \wedge B^{*} \rightarrow \bigotimes_{p} \wedge B^{*}$ also denoted $\bar{\omega}_{x}^{p}$, by setting

$$
\begin{aligned}
\bar{\omega}_{x}^{p}\left(\beta_{1}\right. & \left.\otimes \cdots \otimes \beta_{p}\right) \\
& =\bar{\otimes}_{p}\left[\sum_{f}\left[\sum_{s_{1}<t_{1}} \Delta^{p-1}\left(s_{1}, \ldots, s_{p}\right)(x) \otimes \beta_{1} \otimes \cdots \otimes \beta_{p}\right] .\right.
\end{aligned}
$$

If $x \in \wedge^{\prime} A$, we observe that

$$
\begin{aligned}
& \begin{aligned}
\bar{\omega}_{x}^{p}\left(\beta_{1} \otimes\right. & \left.\cdots \otimes \beta_{p} \otimes y\right) \\
& =\sum_{i}(-1)^{t_{1} \cdot r_{i}} x_{i}\left(\beta_{1}\right) \otimes \bar{\omega}_{x_{i}}^{p-1}\left(\beta_{2} \otimes \cdots \otimes \beta_{p} \otimes y\right) \\
& =\sum_{i}(-1)^{|t| r_{i}} \bar{\omega}_{x_{i}}^{k}\left(\beta_{1} \otimes \cdots \otimes \beta_{k}\right) \otimes \bar{\omega}_{x_{i}}^{p-k}\left(\beta_{k+1} \otimes \cdots \otimes \beta_{p} \otimes y\right)
\end{aligned} \\
& \text { where }|t|=t_{1}+\cdots+t_{k}, \Delta(x)=\sum_{i} x_{i} \otimes x_{i}^{\prime} \text {, and } r_{i}^{\prime}=\operatorname{deg} x_{i}^{\prime} .
\end{aligned}
$$

Lemma 3.1. $\bar{\omega}_{x}^{p} \circ \bar{\omega}_{y}^{p}=\bar{\omega}_{x \wedge y}^{p}$.

Proof. By induction on $p$. Suppose that $x \in \wedge^{r} A, y \in \wedge^{3} A, \beta_{i} \in \wedge^{\beta^{*}} B^{*}$ and $z \in \wedge A$. Then

$$
\begin{aligned}
\bar{\omega}_{x}^{1} \circ \bar{\omega}_{y}^{1}\left(\beta_{1} \otimes z\right) & =\bar{\omega}_{x}^{1}\left(\sum_{j}(-1)^{i, s_{j}^{\prime}} y_{j}\left(\beta_{1}\right) \otimes y_{j}^{\prime} \wedge z\right) \\
& =\sum_{i, j}(-1)^{i s_{j}^{\prime}+\left(i_{1}-s_{j}\right) r_{i}^{\prime}} x_{i}\left(y_{j}\left(\beta_{1}\right)\right) \otimes\left(x_{i}^{\prime} \wedge y_{j}^{\prime} \wedge z\right) .
\end{aligned}
$$

In order to compute $\bar{\omega}_{x \wedge y}^{1}\left(\beta_{1} \otimes z\right)$ we first find $\Delta(x \wedge y)$. Since $\wedge A$ is a Hopf algebra, 


$$
\begin{aligned}
\Delta(x \wedge y) & =\Delta(x) \wedge \Delta(y)=\left(\sum_{i} x_{i} \otimes x_{i}^{\prime}\right) \wedge\left(\sum_{j} y_{j} \otimes y_{j}^{\prime}\right) \\
& =\sum_{i, j}(-1)^{r^{\prime s j}}\left(x_{i} \wedge y_{j}\right) \otimes\left(x_{i}^{\prime} \wedge y_{j}^{\prime}\right) .
\end{aligned}
$$

Hence

$$
\bar{\omega}_{x \wedge y}^{\prime}\left(\beta_{1} \otimes z\right)=\sum_{i, j}(-1)^{s r_{i}+l_{1}\left(r_{i}+s_{j}\right)}\left(x_{i} \wedge y_{j}\right)\left(\beta_{1}\right) \otimes\left(x_{i}^{\prime} \wedge y_{j}^{\prime} \wedge z\right) .
$$

Since $\left(x_{i} \wedge y_{j}\right)\left(\beta_{1}\right)=x_{i}\left(y_{j}\left(\beta_{1}\right)\right)$ and the signs agree, $\bar{\omega}_{x}^{1} \circ \bar{\omega}_{y}^{1}=\bar{\omega}_{x \wedge y}^{1}$.

Now make the induction assumption that $\bar{\omega}_{x}^{k} \circ \bar{\omega}_{y}^{k}=\bar{\omega}_{x \wedge y}^{k}$ for all $k<p$, and consider $\bar{\omega}_{x}^{p} \circ \bar{\omega}_{y}^{p}$.

$$
\begin{aligned}
& \bar{\omega}_{x}^{p} \circ \bar{\omega}_{y}^{p}\left(\beta_{1} \otimes \cdots \otimes \beta_{p} \otimes y\right) \\
&= \bar{\omega}_{x}^{p}\left(\sum_{j}(-1)^{l_{1} s_{j}} y_{j}\left(\beta_{1}\right) \otimes \bar{\omega}_{y j}^{p-1}\left(\beta_{2} \otimes \cdots \otimes \beta_{p} \otimes z\right)\right) \\
&= \sum_{i, j}(-1)^{i, s_{j}+\left(i_{1}-s_{j}\right) r_{i} i} x_{i}\left(y_{j}\left(\beta_{1}\right)\right) \\
& \otimes \bar{\omega}_{x_{1}}^{p-1}\left(\bar{\omega}_{y j}^{p-1}\left(\beta_{2} \otimes \cdots \otimes \beta_{p} \otimes z\right)\right)
\end{aligned}
$$

which by the induction assumption

$$
\begin{aligned}
& =\sum_{i, j}(-1)^{i, s_{j}+i_{1} r_{i}-s j_{i}}\left(x_{i} \wedge y_{j}\right)\left(\beta_{1}\right) \otimes \bar{\omega}_{x_{1} \wedge y_{j}}^{p-1}\left(\beta_{2} \otimes \cdots \otimes \beta_{p} \otimes z\right) \\
& =\bar{\omega}_{x \wedge y}^{p}\left(\beta_{1} \otimes \cdots \otimes \beta_{p} \otimes z\right) .
\end{aligned}
$$

Thus $\bar{\omega}_{x}^{p} \circ \bar{\omega}_{y}^{p}=\bar{\omega}_{x \wedge y}^{p}$ for an arbitrary $p$ completing the proof.

Definition. Given a basis $\xi_{1}, \ldots, \xi_{n}$ for $R^{n *}$ and the map $f: R^{m} \rightarrow R^{n}, m \geq n$, we define

$$
*: K_{p+1}\left(\Lambda^{s} f\right) \otimes K_{q+1}\left(\Lambda^{\prime} f\right) \rightarrow K_{p+q+2}\left(\Lambda^{s} f\right)
$$

for the pairs $p, q$ with $0 \leq p, q \leq m-n$, and $s, t$ with $1 \leq s, t \leq n$, as follows:

Define $*$ for decomposable elements $\gamma=\beta_{1} \otimes \cdots \otimes \beta_{p} \otimes x \in K_{p+1}\left(\wedge^{s} f\right)$ and $\gamma^{\prime}=\beta_{1}^{\prime} \otimes \cdots \otimes \beta_{q}^{\prime} \otimes y \in K_{q+1}\left(\wedge^{\prime} f\right)$ by

$$
\gamma * \gamma^{\prime}=\beta_{1} \otimes \cdots \otimes \beta_{p} \otimes \bar{\omega}_{x}^{q+1}\left(\xi \otimes \beta_{1}^{\prime} \otimes \cdots \otimes \beta_{q}^{\prime} \otimes y\right)
$$

where $\xi=\xi_{1} \wedge \cdots \wedge \xi_{n} \in \wedge^{n} R^{n *}$. Extend * linearly to all of $K_{p+1}\left(\wedge^{s} f\right)$ $\otimes K_{q+1}\left(\wedge^{\prime} f\right)$.

Proposition 3.2. * is associative.

Proof. Let $\gamma$ and $\gamma^{\prime}$ be as in the preceding definition and consider $\gamma^{\prime \prime}$ $=\beta_{1}^{\prime \prime} \otimes \cdots \otimes \beta_{r}^{\prime \prime} \otimes z \in K_{r+1}\left(\Lambda^{\prime \prime} f\right)$. Suppose that $\beta_{i} \in \wedge^{4} R^{n *}, \beta_{i}^{\prime} \in \wedge^{i} R^{n *}$, and $\beta^{\prime \prime}{ }_{i} \in \wedge^{\prime \prime} R^{n *}$. We will show that $\left(\gamma * \gamma^{\prime}\right) * \gamma^{\prime \prime}=\gamma *\left(\gamma^{\prime} * \gamma^{\prime \prime}\right) \in$ $K_{p+q+r+3}\left(\wedge^{s} f\right)$. By definition, 


$$
\begin{gathered}
\left(\gamma * \gamma^{\prime}\right) * \gamma^{\prime \prime}=\left[\beta_{1} \otimes \cdots \otimes \beta_{p} \otimes \bar{\omega}_{x}^{q+1}\left(\xi \otimes \beta_{1}^{\prime} \otimes \cdots \otimes \beta_{q}^{\prime} \otimes y\right)\right] * \gamma^{\prime \prime} \\
=\left[\beta_{1} \otimes \cdots \otimes \beta_{p} \otimes \sum_{i}(-1)^{\left|t^{\prime}\right| \operatorname{deg} x_{i}} \bar{\omega}_{x_{1}}^{q+1}\left(\xi \otimes \beta_{1}^{\prime} \otimes \cdots \otimes \beta_{q}^{\prime}\right)\right. \\
\left.\otimes\left(x_{i}^{\prime} \wedge y\right)\right] * \gamma^{\prime \prime} \\
\text { where }\left|t^{\prime}\right|=t_{1}^{\prime}+\cdots+t_{q}^{\prime}+n, \\
=\sum_{i}(-1)^{\left|r^{\prime}\right| \operatorname{deg} x_{1}} \beta_{1} \otimes \cdots \otimes \beta_{p} \otimes \bar{\omega}_{x_{1}}^{q+1}\left(\xi \otimes \beta_{1}^{\prime} \otimes \cdots \otimes \beta_{q}^{\prime}\right) \\
\otimes \bar{\omega}_{x_{i} \wedge y}^{r+1}\left(\xi \otimes \gamma^{\prime \prime}\right) .
\end{gathered}
$$

On the other hand,

$$
\begin{aligned}
\gamma *\left(\gamma^{\prime} *\right. & \left.\gamma^{\prime \prime}\right) \\
= & \beta_{1} \otimes \cdots \otimes \beta_{p} \\
& \otimes \bar{\omega}_{x}^{q+r+2}\left(\xi \otimes \beta_{1}^{\prime} \otimes \cdots \otimes \beta_{q}^{\prime} \otimes \bar{\omega}_{y}^{r+1}\left(\xi \otimes \gamma^{\prime \prime}\right)\right) \\
= & \sum_{i}(-1)^{\left|t^{\prime}\right| \operatorname{deg} x_{i}^{\prime}} \beta_{1} \otimes \cdots \otimes \beta_{p} \otimes \bar{\omega}_{x_{1}}^{q+1}\left(\xi \otimes \beta_{1}^{\prime} \otimes \cdots \otimes \beta_{q}^{\prime}\right) \\
& \otimes \bar{\omega}_{x_{i}^{r+1}}^{r+1}\left(\bar{\omega}_{y}^{r+1}\left(\xi \otimes \gamma^{\prime \prime}\right)\right)
\end{aligned}
$$

which by Lemma 3.1 is seen to equal $\left(\gamma * \gamma^{\prime}\right) * \gamma^{\prime \prime}$.

Lemma 3.3 (Buchsbaum [3]). Consider $\lambda, x, y \in \wedge A$ and $\beta \in \wedge A^{*}$ and let $\Delta(\lambda)=\sum \lambda_{i} \otimes \lambda_{i}^{\prime}, \Delta(x)=\sum x_{j} \otimes x_{j}^{\prime}$, and $\Delta^{*}(\beta)=\sum \beta_{k} \otimes \beta_{k}^{\prime}$. Then the following formulas hold:

(1) $\beta(x \wedge y)=\Sigma_{k}(-1)^{\operatorname{deg} x \cdot \operatorname{deg} \beta_{k}^{\prime}} \beta_{k}(x) \wedge \beta_{k}^{\prime}(y)$.

(2) $\Delta(\beta(x))=\sum_{j} \beta\left(x_{j}\right) \otimes x_{j}^{\prime}=\sum_{j}(-1)^{\operatorname{deg} x_{j} \operatorname{deg} \beta} x_{j} \otimes \beta\left(x_{j}^{\prime}\right)$.

(3) $\lambda(\beta)(x)=\sum_{i}(-1)^{\operatorname{deg} \lambda_{i}^{\prime} \operatorname{deg} \beta} \lambda_{i} \wedge \beta\left(\lambda_{i}^{\prime} \wedge x\right)$.

The next lemma will be used to show that * satisfies the Leibniz formula.

Lemma 3.4. Consider $f: R^{m} \rightarrow R^{n}, m \geq n$, and let $\xi, \gamma$, and $\gamma^{\prime}$ be as in the definition preceding Proposition 3.2. Then

$$
\begin{gathered}
\sum_{i}(-1)^{n \cdot \operatorname{deg} x_{i}^{\prime}} \beta_{1} \otimes \cdots \otimes \beta_{p} \otimes x_{i}(\xi) \otimes \bar{\omega}_{x_{i}^{q}}^{q-1}\left(d\left(\gamma^{\prime}\right)\right) \\
=\sum_{i}(-1)^{n \cdot \operatorname{deg} x_{i}^{\prime}} \beta_{1} \otimes \cdots \otimes \beta_{p} \otimes x_{i}(\xi) \otimes d\left(\bar{\omega}_{x_{i}}^{q}\left(\gamma^{\prime}\right)\right) \\
+\sum_{i}(-1)^{n n_{1}^{\prime}+\left(n+r_{i}\right) \cdot \operatorname{deg} x_{i}^{\prime}} \beta_{1} \otimes \cdots \otimes \beta_{p} \otimes x_{i}(\xi) \\
\otimes x_{i, l_{i}^{\prime}}^{\prime}\left(\beta_{1}^{\prime}\right) \cdot \bar{\omega}_{x_{i}^{\prime}}^{q-1}\left(\beta_{2}^{\prime} \otimes \cdots \otimes \beta_{q}^{\prime} \otimes y\right)
\end{gathered}
$$

where as a special case of the map $\Delta^{p}\left(n_{1}, \ldots, n_{p+1}\right)$,

$$
\Delta^{2}\left(, t_{1}^{\prime},-\right)(x)=\sum_{i} x_{i} \otimes x_{i, t_{i}^{\prime}}^{\prime} \otimes x_{i}^{\prime \prime}
$$


with $\operatorname{deg} x_{i, r_{1}^{\prime}}^{\prime}=t_{1}^{\prime}$ and so $x_{i, t_{1}^{\prime}}^{\prime}\left(\beta_{1}^{\prime}\right)$ is a scalar.

Proof. The proof follows immediately from Lemma 2.4 of [3] and the definition of $\bar{\omega}_{x}^{q}$.

Proposition 3.5. With hypotheses as in Lemma 3.4,

$$
d\left(\gamma * \gamma^{\prime}\right)=d(\gamma) * \gamma^{\prime}+(-1)^{p+1} \gamma * d\left(\gamma^{\prime}\right)
$$

Proof.

$$
\begin{aligned}
& d(\gamma) * \gamma^{\prime}+(-1)^{p+1} \gamma * d\left(\gamma^{\prime}\right) \\
& =\left[\sum_{j=1}^{p-1}(-1)^{j+1} \beta_{1} \otimes \cdots \otimes \beta_{j} \wedge \beta_{j+1} \otimes \cdots \otimes \beta_{p} \otimes x\right] * \gamma^{\prime} \\
& +(-1)^{p+1}\left(\beta_{1} \otimes \cdots \otimes \beta_{p-1} \otimes \beta_{p}(x)\right) * \gamma^{\prime}+(-1)^{p+1} \gamma * d\left(\gamma^{\prime}\right) \\
& =\sum_{j=1}^{p-1}(-1)^{j+1} \beta_{1} \otimes \cdots \otimes \beta_{j} \wedge \beta_{j+1} \otimes \cdots \otimes \beta_{p} \otimes \bar{\omega}_{x}^{q+1}\left(\xi \otimes \gamma^{\prime}\right) \\
& +(-1)^{p+1} \beta_{1} \otimes \cdots \otimes \beta_{p-1} \otimes \bar{\omega}_{\beta,(x)}^{q+1}\left(\xi \otimes \gamma^{\prime}\right)+(-1)^{p+1} \gamma * d\left(\gamma^{\prime}\right) \\
& {\left[\begin{array}{rl}
= & \sum_{j=1}^{p-1}(-1)^{j+1} \beta_{1} \otimes \cdots \otimes \beta_{j} \wedge \beta_{j+1} \otimes \cdots \otimes \beta_{p} \otimes \bar{\omega}_{x}^{q+1}\left(\xi \otimes \gamma^{\prime}\right) \\
& +(-1)^{p+1} \sum_{i}(-1)^{n \cdot \operatorname{deg} x_{i}^{\prime}} \beta_{1} \otimes \cdots \otimes \beta_{p-1} \otimes \beta_{p}\left(x_{i}\right)(\xi) \otimes \bar{\omega}_{x_{i}}^{q}\left(\gamma^{\prime}\right) \\
& +(-1)^{p+1} \beta_{1} \otimes \cdots \otimes \beta_{p} \otimes \bar{\omega}_{x}^{q}\left(\xi \otimes d\left(\gamma^{\prime}\right)\right),
\end{array}\right.}
\end{aligned}
$$

where to obtain the middle sum of (1) we use Lemma 3.3(2) to find that $\Delta\left(\beta_{p}(x)\right)=\sum_{i} \beta_{p}\left(x_{i}\right) \otimes x_{i}^{\prime}$. We can further simplify the terms of this sum by using Lemma 3.3(3) as follows:

$$
\beta_{p}\left(x_{i}\right)(\xi)=\sum_{j}(-1)^{\operatorname{deg} \beta_{N}^{\prime} \cdot \operatorname{deg} x_{i}} \beta_{p j} \wedge x_{i}\left(\beta_{p j}^{\prime} \wedge \xi\right) .
$$

Since $\operatorname{deg} \xi=n$, all terms in the expansion of $\beta_{p}\left(x_{i}\right)(\xi)$ are zero except those in which $\operatorname{deg} \beta_{p j}^{\prime}=0$, and since $\wedge R^{n *}$ is a Hopf algebra, the only such term is the one with $\beta_{p j}=\beta_{p}$ and $\beta_{p j}^{\prime}=1$. Consequently,

$$
\beta_{p}\left(x_{i}\right)(\xi)=\beta_{p} \wedge x_{i}(\xi)
$$

where $x_{i}(\xi)$ might be a scalar. We can therefore rewrite the sums of (1) as (A), (B), and (C) where

$$
\begin{aligned}
& (\mathrm{A})=\sum_{j=1}^{p-1}(-1)^{j+1} \beta_{1} \otimes \cdots \otimes \beta_{j} \wedge \beta_{j+1} \otimes \cdots \otimes \beta_{p} \otimes \bar{\omega}_{x}^{q+1}\left(\xi \otimes \gamma^{\prime}\right), \\
& (\mathrm{B})=(-1)^{p+1} \sum_{i}(-1)^{n \cdot \operatorname{deg} x_{i}^{\prime}} \beta_{1} \otimes \cdots \otimes \beta_{p-1} \otimes \beta_{p} \wedge x_{i}(\xi) \otimes \bar{\omega}_{x_{i}^{q}}^{q}\left(\gamma^{\prime}\right),
\end{aligned}
$$


and

$$
\text { (C) }=(-1)^{p+1} \sum_{i}(-1)^{n \cdot \operatorname{deg} x_{i}^{\prime}} \beta_{1} \otimes \cdots \otimes \beta_{p} \otimes x_{i}(\xi) \otimes \bar{\omega}_{x_{i}}^{q-1}\left(d\left(\gamma^{\prime}\right)\right),
$$

and where (B) includes terms with $x_{i}(\xi)$ a scalar but (C) does not.

On the other hand,

$$
\begin{aligned}
& d\left(\gamma * \gamma^{\prime}\right)=d\left(\beta_{1} \otimes \cdots \otimes \beta_{p} \otimes \bar{\omega}_{x}^{q+1}\left(\xi \otimes \gamma^{\prime}\right)\right) \\
& =d\left[\sum_{i}(-1)^{n \cdot \operatorname{deg} x_{i}^{\prime}} \beta_{1} \otimes \cdots \otimes \beta_{p} \otimes x_{i}(\xi) \otimes \bar{\omega}_{x_{i}}^{q}\left(\gamma^{\prime}\right)\right] \\
& =d\left[\sum_{i}(-1)^{n \cdot \operatorname{deg} x_{i}^{\prime}+\left(n+t_{i}^{\prime}\right) \operatorname{deg} x_{i}^{\prime \prime}} \beta_{1} \otimes \cdots \otimes \beta_{p} \otimes x_{i}(\xi) \otimes x_{i}^{\prime}\left(\beta_{1}^{\prime}\right)\right. \\
& \left.\otimes \bar{\omega}_{x_{i}}^{q-1}\left(\beta_{2}^{\prime} \otimes \cdots \otimes \beta_{q}^{\prime} \otimes y\right)\right] \\
& =\sum_{j=1}^{p-1}(-1)^{j+1} \beta_{1} \otimes \cdots \otimes \beta_{j} \wedge \beta_{j+1} \otimes \cdots \otimes \beta_{p} \otimes \bar{\omega}_{x}^{q+1}\left(\xi \otimes \gamma^{\prime}\right) \\
& +(-1)^{p+1} \sum_{i}(-1)^{n-\operatorname{deg} x_{i}^{\prime}} \beta_{1} \otimes \cdots \otimes \beta_{p} \wedge x_{i}(\xi) \otimes \bar{\omega}_{x_{i}}^{q}\left(\gamma^{\prime}\right) \\
& +(-1)^{p+2} \sum_{i}(-1)^{n-\operatorname{deg} x_{i}^{\prime}+\left(n+t_{1}^{\prime}\right) \operatorname{deg} x_{1}^{\prime \prime}} \beta_{1} \otimes \cdots \otimes \beta_{p} \otimes x_{i}(\xi) \wedge x_{i}^{\prime}\left(\beta_{1}^{\prime}\right) \\
& \otimes \bar{\omega}_{x, 1}^{q-1}\left(\beta_{2}^{\prime} \otimes \cdots \otimes \beta_{q}^{\prime} \otimes y\right) \\
& +(-1)^{p+3} \sum_{i}(-1)^{n \cdot \operatorname{deg} x_{i}} \beta_{1} \otimes \cdots \otimes \beta_{p} \otimes x_{i}(\xi) \otimes d\left(\bar{\omega}_{x_{i}}^{q}\left(\gamma^{\prime}\right)\right) \text {. }
\end{aligned}
$$

Let (a), (b), (c), and (d) be the respective sums of (2). Observe that (b) does not contain terms where $x_{i}(\xi)$ is a scalar nor does $(c)$ include terms where either $x_{i}(\xi)$ or $x_{i}^{\prime}\left(\beta_{1}^{\prime}\right)$ is a scalar.

We must show that $(1)=(2)$. Comparing corresponding component sums we see that $(A)=(a)$ and $(B)=(b)+\left(b^{\prime}\right)$ where

$$
\left(b^{\prime}\right)=(-1)^{p+1} \sum_{i}(-1)^{n \cdot \operatorname{deg} x_{i}^{i}} \beta_{1} \otimes \cdots \otimes \beta_{p-1} \otimes \beta_{p} \cdot x_{i, n}(\xi) \otimes \bar{\omega}_{x_{i}}^{q}\left(\gamma^{\prime}\right)
$$

and $x_{i, n}(\xi)$ is a scalar. Before considering the other terms, observe that Lemma 3.3(1) together with the fact that $\xi \wedge \beta_{\mathrm{i}}^{\prime}=0$ imply

$$
\begin{aligned}
\sum_{\operatorname{deg} x_{i}<n ; \operatorname{deg} x_{i}<t_{1}^{\prime}} & (-1)^{n-\operatorname{deg} x_{i}} x_{i}(\xi) \wedge x_{i}^{\prime}\left(\beta_{1}^{\prime}\right) \\
& =-\sum_{i}(-1)^{n \cdot \operatorname{deg} x_{i}} x_{i, n}(\xi) \cdot x_{i}^{\prime}\left(\beta_{1}^{\prime}\right)-(-1)^{n t_{i}^{\prime}} \sum_{i} x_{i}(\xi) \cdot x_{i, j_{i}^{\prime}}^{\prime}\left(\beta_{1}^{\prime}\right) .
\end{aligned}
$$

Since (c) does not contain terms in which $x_{i}(\xi)$ or $x_{i}^{\prime}\left(\beta_{1}^{\prime}\right)$ are scalars, (3) implies that (c) can be rewritten as 


$$
\begin{gathered}
(-1)^{p+1} \sum_{i}(-1)^{n-\operatorname{deg} x_{i}^{\prime}+\left(n+t_{i}\right) \cdot \operatorname{deg} x_{i}^{\prime \prime}} \beta_{1} \otimes \otimes \beta_{p} \otimes x_{i, n}(\xi) \cdot x_{i}^{\prime}\left(\beta_{1}^{\prime}\right) \\
\otimes \bar{\omega}_{x_{i}^{q}}^{q-1}\left(\beta_{2}^{\prime} \otimes \cdots \otimes \beta_{q}^{\prime} \otimes y\right) \\
+(-1)^{p+1} \sum_{i}(-1)^{n t_{i}+\left(n+r_{i}\right) \cdot \operatorname{deg} x_{i}^{\prime \prime}} \beta_{1} \otimes \cdots \otimes \beta_{p} \otimes x_{i}(\xi) \cdot x_{i, s_{i}^{\prime}}^{\prime}\left(\beta_{1}^{\prime}\right) \\
\otimes \bar{\omega}_{x_{i}^{q}-1}^{q-1}\left(\beta_{2}^{\prime} \otimes \cdots \otimes \beta_{q}^{\prime} \otimes y\right) .
\end{gathered}
$$

We can therefore write $(c)=\left(b^{\prime}\right)+\left(c^{\prime}\right)$ where $\left(c^{\prime}\right)$ is the sum immediately above. By Lemma 3.4, (C) $=\left(c^{\prime}\right)+(d)$ and so we have

$$
\begin{aligned}
(A)+(B)+(C) & =(a)+(b)+\left(b^{\prime}\right)+\left(c^{\prime}\right)+(d) \\
& =(a)+(b)+(c)+(d)
\end{aligned}
$$

which shows that $(1)=(2)$ and completes the proof of the proposition.

We can now prove the first main result of this section.

Theorem 3.6. Consider $f: R^{m} \rightarrow R^{n}, m \geq n$, and let $\xi_{1}, \ldots, \xi_{n}$ be a basis for $R^{n *}$. Then the maps * induce on $K\left(\wedge^{n} f\right)$ the structure of a differential graded $R$-algebra. The maps * moreover induce on each complex $K\left(\wedge^{p} f\right), 1 \leq p \leq n$, the structure of $a\left(\right.$ right) differential graded $K\left(\wedge^{n} f\right)$-module.

Proof. The major parts of the proof, namely that * is associative, satisfies the Leibniz formula and that $K\left(\wedge^{p} f\right) * K\left(\wedge^{n} f\right) \subset K\left(\wedge^{p} f\right)$, follow from Propositions 3.2 and 3.5 and the definition of the map *. We need only define * in the lowest dimensions and check that it behaves properly.

If $b \in \wedge^{p} R^{n}=K_{0}\left(\wedge^{p} f\right)$ and $\gamma=\beta_{1} \otimes \cdots \otimes \beta_{q} \otimes x \in K_{q+1}\left(\wedge^{n} f\right), q \geq 0$, we define $b * \gamma \in K_{q+1}\left(\wedge^{p} f\right)$ by

$$
b * \gamma=b(\xi) \wedge \beta_{1} \otimes \beta_{2} \otimes \cdots \otimes \beta_{p} \otimes x .
$$

On the other hand, if $b \in \wedge^{n} R^{n}=K_{0}\left(\wedge^{n} f\right)$ and $\gamma \in K_{q}\left(\wedge^{p} f\right)$ with $q \geq 0$ and $n \geq p$, we define $\gamma * b=b * \gamma \in K_{q}\left(\wedge^{p} f\right)$ by

$$
\gamma * b=(-1)^{\alpha(n)} \gamma \cdot b(\xi)=(-1)^{o(n)} b(\xi) \cdot \gamma=b * \gamma
$$

where $\sigma(n)=\frac{1}{2} n(n+3)$. We observe that if $e_{1}, \ldots, e_{n}$ is the basis for $R^{n}$ such that $\xi_{1}, \ldots, \xi_{n}$ is its dual basis and $e=e_{1} \wedge \cdots \wedge e_{n}$, then $e(\xi)=\xi(e)$ $=(-1)^{\sigma(n)}$ and so the map from $\wedge^{n} R^{n}$ to $R$ defined by $b \mapsto(-1)^{o(n)} b(\xi)$ is precisely the map $e \mapsto 1$. The inverse of this map defines the unit $e$ of the algebra $K\left(\Lambda^{n} f\right)$ in such a way that $e * \gamma=\gamma=\gamma * e$ making $K\left(\Lambda^{p} f\right)$ a unitary $K\left(\wedge^{n} f\right)$-module.

A straightforward check now shows that for any $\gamma \in K_{r}\left(\wedge^{p} f\right), \gamma^{\prime} \in K_{s}\left(\wedge^{n} f\right)$, and $\gamma^{\prime \prime} \in K_{t}\left(\wedge^{n} f\right)$ with $1 \leq p \leq n$ and $r, s, t \geq 0$,

$$
\left(\gamma * \gamma^{\prime}\right) * \gamma^{\prime \prime}=\gamma *\left(\gamma^{\prime} * \gamma^{\prime \prime}\right), \quad d\left(\gamma * \gamma^{\prime}\right)=d(\gamma) * \gamma^{\prime}+(-1)^{\prime} \gamma * d\left(\gamma^{\prime}\right)
$$

and this completes the proof of the theorem. 
As an immediate consequence we obtain

Corollary 3.7 (Theorem 1.1, part (a)). If $f: R^{m} \rightarrow R^{n}, m \geq n$, then

\section{Ann Coker $\wedge^{n} f \subset$ Ann Coker $H\left(K\left(\wedge^{p} f\right)\right)$}

for each $p, 1 \leq p \leq n$.

Proof. The proof follows immediately from Theorem 3.6 and the observation that Ann Coker $\wedge^{n} f \approx B_{0}\left(K\left(\Lambda^{n} f\right)\right)=$ the zero-dimensional boundaries of $K\left(\wedge^{n} f\right)$.

Notation. Given a complex $(K, d)$, we will let $B(K)=\operatorname{Im} d, Z(K)=\operatorname{Ker} d$, and $H(K)=Z(K) / B(K)$. If $z$ is a cycle with homology class [z], we write $z \in[z] \in H(K)$.

Lemma 3.8. Suppose $f: R^{m} \rightarrow R^{n}, m \geq n>1$, is the zero map. Consider $\gamma_{i} \in K_{p_{i}}\left(\Lambda^{n} f\right)$ and $\gamma_{i}^{\prime} \in K_{q_{i}}\left(\Lambda^{n} f\right)$ with $i=1, \ldots$, s and where $p_{i}, q_{i}>0$ are such that $p_{i}+q_{i}=p+q$ for each $i$ and

$$
z=\sum_{i=1}^{s} \gamma_{i} * \gamma_{i}^{\prime} \in Z_{p+q}\left(K\left(\wedge^{n} f\right)\right)
$$

Then $[z]=0 \in H_{p+q}\left(K\left(\wedge^{n} f\right)\right)$.

Proof. We shall show that $z$ is homologous to a cycle $z^{n} \in Z_{p+q}\left(\bar{K}\left(\wedge^{n} f\right)\right)$ of the form

$$
z^{\prime \prime}=\sum_{j} \bar{\gamma}_{j}^{\prime \prime} \otimes \beta_{j}^{\prime \prime}\left(x_{j}\right)
$$

where $\bar{\gamma}_{j}^{\prime \prime} \in \Theta_{p+q-1} R^{n *}$ and $\beta_{j}^{n} \in \Lambda^{*} R^{n *}$ with $t_{j}>0$. Then, since $f=0$, it follows that $\omega_{f^{\circ}}=0$ and so $\beta_{j}^{\prime \prime}(x)=0$ for each $j$. Hence $z^{\prime \prime}=0$ which will show that $[z]=0$ and complete the proof.

We may assume without loss of generality that $\gamma_{i}$ and $\gamma_{i}^{\prime}$ are decomposable elements. Therefore we may write $\gamma_{i}=\bar{\gamma}_{i} \otimes x_{i}$, where $\bar{\gamma}_{i} \in \otimes_{k=1}^{p_{i}-1} \wedge^{s_{k}} R^{n *}$ and $x_{i} \in \wedge^{n+\sum s_{k}} R^{m}$, and express $\gamma_{i}^{\prime}$ in a similar fashion. It follows that

$$
\gamma_{i} * \gamma_{i}^{\prime}=\bar{\gamma}_{i} \otimes \bar{\omega}_{x_{i}}^{q}\left(\xi \otimes \bar{\gamma}_{i}^{\prime} \otimes y_{i}\right)=\sum_{k} \pm \bar{\gamma}_{i} \otimes \bar{\omega}_{x_{k}}^{q}\left(\xi \otimes \bar{\gamma}_{i}^{\prime}\right) \otimes x_{i k}^{\prime} \wedge y_{i} .
$$

But $f=0$ implies that $\omega_{f}=0$, and so $\bar{\omega}_{x_{k k}}^{q}\left(\xi \otimes \bar{\gamma}_{i}^{\prime}\right)=0$ as well for all $x_{i k}$ such that $\operatorname{deg} x_{i k}>0$. Consequently,

$$
\gamma_{i} * \gamma_{i}^{\prime}= \pm \bar{\gamma}_{i} \otimes \xi \otimes \bar{\gamma}_{i}^{\prime} \otimes x_{i} \wedge y_{i} \notin \bar{K}_{p+q}\left(\wedge^{n} f\right)
$$

which shows that

$$
z=\sum_{i} \gamma_{i} * \gamma_{i}^{\prime} \notin R_{p+q}\left(\Lambda^{n} f\right)
$$


However, by Corollary $2.5, z$ is homologous to some cycle $z^{\prime \prime} \in \bar{K}_{p+q}\left(\wedge^{n} f\right)$ and so there exists $w \in K_{p+q+1}\left(\bigwedge^{n} f\right)$ such that $d(w)=z-z^{\prime \prime}$. By the Remark 2.4, we may write

$$
K_{p+q+1}\left(\bigwedge^{n} f\right)=\bar{K}_{p+q+1}\left(\bigwedge^{n} f\right) \oplus Y_{p+q+1}
$$

which means $w$ is expressible in the form $w_{1}+w_{2}$ where $w_{1} \in \bar{K}_{p+q+1}\left(\wedge^{n} f\right)$ is the symmetric part of $w$ of lowest possible degree and $w_{2} \in Y_{p+q+1}$. Now $d\left(w_{1}\right)$ $\in B_{p+q}\left(\bar{K}\left(\wedge^{n} f\right)\right)$ must be of the form $\sum_{k} \bar{\gamma}_{k}^{\prime \prime} \otimes \beta_{k}^{\prime \prime}\left(x_{k}\right)$ where $\bar{\gamma}_{k}^{\prime \prime} \in \Theta_{p+q-1} R^{n *}$, $\operatorname{deg} \beta_{k}^{\prime \prime}>0$, and $x_{k} \in \wedge^{n+p+q-1} R^{m}$. Moreover, $d\left(w_{2}\right)$ must be of the form $y+\Sigma_{l} \bar{\gamma}_{l}^{\prime \prime} \otimes \beta_{l}^{\prime \prime}\left(x_{l}\right)$ where $y \in Y_{p+q}$ and the sum is in $B_{p+q}\left(\bar{K}\left(\wedge^{n} f\right)\right)$. Therefore,

$$
\begin{aligned}
z^{\prime \prime} & =z-d(w)=z-d\left(w_{2}\right)-d\left(w_{1}\right) \\
& =(z-y)-\sum_{l} \bar{\gamma}_{l}^{\prime \prime} \otimes \beta_{l}^{\prime \prime}\left(x_{l}\right)-\sum_{k} \gamma_{k}^{\prime \prime} \otimes \beta_{k}^{\prime \prime}\left(x_{k}\right) \\
& =(z-y)-\sum_{j} \bar{\gamma}_{j} \otimes \beta_{j}^{\prime \prime}\left(x_{j}\right),
\end{aligned}
$$

and since $\bar{K}_{p+q}\left(\wedge^{n} f\right) \cap Y_{p+q}=0$, we must have $z-y=0$ which establishes (1) and completes the proof.

The second main result of this section now follows.

Theorem 3.9. If $f: R^{m} \rightarrow R^{n}, m \geq n>1$, is the zero map, then $H\left(K\left(\wedge^{n} f\right)\right)$ has trivial Massey operations. In particular, $H\left(K\left(\Lambda^{n} f\right)\right)$ has trivial multiplicative structure.

Remark. The treatment of Massey operations given here is based upon the description given by Gulliksen and Levin in [10].

Proof. We give $H\left(K\left(\wedge^{n} f\right)\right)$ the algebra structure induced by *. Consider $\gamma \in[\gamma] \in H_{p}\left(K\left(\wedge^{n} f\right)\right)$ and $\gamma^{\prime} \in\left[\gamma^{\prime}\right] \in H_{q}\left(K\left(\wedge^{n} f\right)\right)$ where $p, q>0$. By the preceding lemma, $[\gamma] *\left[\gamma^{\prime}\right]=\left[\gamma * \gamma^{\prime}\right]=0$ which shows that $H\left(K\left(\wedge^{n} f\right)\right)$ has trivial multiplication for elements of positive degree.

Now let $H=\sum_{t>0} H_{t}\left(K\left(\wedge^{n} f\right)\right)$. The second order Massey operation[M(_, $\left.)\right]$ : $H \otimes H \rightarrow H$ is defined for $[\gamma],\left[\gamma^{\prime}\right] \in H$ by $\left[M\left(\gamma, \gamma^{\prime}\right)\right]=\left[\gamma * \gamma^{\prime}\right]$. Since the multiplication is trivial on $H,\left[M\left({ }_{-}\right)\right]=0$. Given $\left[\gamma_{1}\right],\left[\gamma_{2}\right],\left[\gamma_{3}\right] \in H$, the ternary Massey operation $[M(,,-)]:, H \otimes H \otimes H \rightarrow H$ is a well-defined function of three homology classes defined as follows:

$$
\left[M\left(\gamma_{1}, \gamma_{2}, \gamma_{3}\right)\right]=\left[\gamma_{1} * \lambda\left(\gamma_{2}, \gamma_{3}\right)+\lambda\left(\gamma_{1}, \gamma_{2}\right) * \gamma_{3}\right]
$$

where, since $\left[M\left({ }_{-}\right)\right]=0, \lambda\left(\gamma_{1}, \gamma_{2}\right), \lambda\left(\gamma_{2}, \gamma_{3}\right) \in K\left(\wedge^{n} f\right)$ can be chosen so that $d \lambda\left(\gamma_{1}, \gamma_{2}\right)=M\left(\gamma_{1}, \gamma_{2}\right)$ and $d \lambda\left(\gamma_{2}, \gamma_{3}\right)=M\left(\gamma_{2}, \gamma_{3}\right)$. Again by Lemma 3.8, $\left[M\left(\gamma_{1}, \gamma_{2}, \gamma_{3}\right)\right]=0$.

Assume now that all $(n-1)$ st order Massey operations are trivial and define $[M(, \ldots)]:, \otimes_{n} H \rightarrow H$ by 


$$
\begin{aligned}
M\left(\gamma_{1}, \ldots, \gamma_{n}\right)= & \gamma_{1} * \lambda\left(\gamma_{2}, \ldots, \gamma_{n}\right) \\
& +\sum_{i=2}^{n-2}(-1)^{\sum_{j=1}^{\prime-1}\left(\operatorname{deg} \gamma_{j}+1\right)} \lambda\left(\gamma_{1}, \ldots, \gamma_{i}\right) * \lambda\left(\gamma_{i+1}, \ldots, \gamma_{n}\right) \\
& +(-1)^{\sum_{j-1}^{n-2}\left(\operatorname{deg} \gamma_{j}+1\right)} \lambda\left(\gamma_{1}, \ldots, \gamma_{n-1}\right) * \gamma_{n}
\end{aligned}
$$

where $\lambda\left(\gamma_{1}, \ldots, \gamma_{i}\right)$ is such that $d \lambda\left(\gamma_{1}, \ldots, \gamma_{i}\right)=M\left(\gamma_{1}, \ldots, \gamma_{i}\right)$. Once again Lemma 3.8 implies that $\left[M\left(\gamma_{1}, \ldots, \gamma_{n}\right)\right]=0$ and so $H$ has trivial $n$th order Massey operations for each $n \geq 2$.

4. The local case. In this section we apply the results of $\$ 3$ to obtain very easily complete information about certain homological invariants of the factor rings of regular local rings called generalized local complete intersections. Throughout this section, $R$ will be a noetherian local ring with maximal ideal $\mathfrak{m}$, residue class field $k=R / \mathfrak{m}$, and $\mathfrak{m}$-adic completion $\hat{R}$. If $x_{1}, \ldots, x_{t}$ minimally generate $\mathfrak{m}$, let $\varphi: R^{t} \rightarrow R$ be the map whose matrix is $\left(x_{1 j}\right)$ with $x_{1 j}=x_{j}$. Then $K(\varphi)$, or simply $K$, will denote the ordinary Koszul complex defined by $\varphi$ and will be called the Koszul complex of $R$.

We refer to [10] for the definitions and elementary properties of the homological invariants $H(K)$, $\operatorname{Tor}^{R}(k, k)$, Betti numbers $b_{i}(R)$, and Poincaré series $P_{R}(z)$. We observe here that a ring $R$ is characterized by any of these invariants if and only if its completion $\hat{R}$ is characterized in the same way. Since $\hat{R}$ is always a factor ring of some regular local ring by the Cohen structure theorem, a characterization of local complete intersections (as defined in [10]), for example, can be given for those rings $R$ which are assumed to be of the form $R=R^{\prime} / a$ where $R^{\prime}$ is regular. Moreover, $R^{\prime}$ noetherian means that $R$ can be taken to be a proper homomorphic image of $R^{\prime}$ in the sense that a $\subset \mathrm{m}^{\prime 2}$. The ring $R=R^{\prime} / \mathfrak{a}$ is then a local complete intersection if and only if a can be generated by an $R^{\prime}$ sequence. If $a$ is minimally generated by $r$ elements and $f: R^{\prime r} \rightarrow R^{\prime}$ is a map such that Coker $f=R$, then $R$ is a local complete intersection if and only if $K(f)$ is acyclic.

The following is a well-known and useful fact.

Proposition 4.1. If $R$ is a proper homomorphic image of regular local ring $R^{\prime}$, then $H(K) \approx \operatorname{Tor}^{R^{\prime}}(R, k)$ as algebras where $K$ is the Koszul complex of $R$.

Remark. Assmus's characterization [1] of local complete intersections as rings for which $H(K)$ is an exterior algebra on $r$ generators, where $R=R^{\prime} / a$ and $a$ is minimally generated by $r$ elements, follows from 4.1 by an argument based upon the behavior of the natural map

$$
\Gamma_{*}: H_{*}\left(K(f) \otimes_{R^{\prime}} k\right) \rightarrow \operatorname{Tor}^{R^{\prime}}(R, k) .
$$

Using the five term exact sequence of low order terms obtained from the Künneth spectral sequence convergent to $\operatorname{Tor}^{R^{\prime}}(R, k)$, one finds that $K(f)$ is 
acyclic if and only if $\Gamma_{2}$ is an epimorphism. But from 4.1, $\Gamma_{2}$ is an epimorphism if and only if $\left(H_{1}(K)\right)^{2}=H_{2}(K)$. Details of the proof can be found independently in $[9,2.19]$ and $[11,10.8]$.

Definition. Let $R^{\prime}$ be a regular local ring and consider a map $f: R^{\prime m} \rightarrow R^{\prime n}$, $m \geq n$, such that $f\left(R^{\prime m}\right) \subset m^{\prime} R^{\prime n}$. Let $I=$ Ann Coker $\wedge^{n} f$. A ring $R$ of the form $R^{\prime} / I$ is a generalized local complete intersection of type $(m, n)$, or briefly a $G L C I$ ring of type $(m, n)$, if and only if $\operatorname{depth}\left(I ; R^{\prime}\right)=m-n+1$, i.e., if and only if $H\left(K\left(\wedge^{n} f\right)\right)$ is acyclic.

Definition. Following [10], a local ring $R$ is a Golod ring if and only if $H(K)$ has trivial Massey operations.

Theorem 4.2. If $R$ is a GLCI ring of type $(m, n)$ with $n>1$, then $R$ is a Golod ring and

$$
c_{i}=\operatorname{dim}_{k} H_{i}(K)=\left(\begin{array}{c}
n+i-2 \\
n-1
\end{array}\right) \cdot\left(\begin{array}{c}
m \\
n+i-1
\end{array}\right) \text { for } i>0 .
$$

Proof. If $R$ is a GLCI ring of type $(m, n)$ then, by $4.1, H(K) \approx \operatorname{Tor}^{R^{\prime}}(R, k)$. But $K\left(\wedge^{n} f\right)$ is an $R^{\prime}$-free resolution of $R$ and so $H(K) \approx H\left(K\left(\wedge^{n} f\right) \otimes_{R^{\prime}} k\right)$ $=H\left(K\left(\wedge^{n} \bar{f}\right)\right)$, where $\bar{f}: k^{m} \rightarrow k^{n}$ is the zero map. Thus $H(K)$ has trivial Massey operations by Theorem 3.9 and so $R$ is a Golod ring.

The formula for $c_{i}=\operatorname{dim}_{k} H_{i}(K)$ follows by using Proposition 2.1 to obtain the isomorphism $H(K) \approx H\left(C\left(\wedge^{n} \bar{f}\right)\right)$. Since $\bar{f}=0$, the boundary map in the complex $C\left(\wedge^{n} \bar{f}\right)$ is also the zero map and so $H\left(C\left(\wedge^{n} \bar{f}\right)\right)=C\left(\wedge^{n} \bar{f}\right)$. For $i>0$, this says that

$$
H_{i}(K) \approx S_{i-1}\left(k^{n *}\right) \otimes \wedge^{n+i-1} k^{m}
$$

which implies that

$$
c_{i}=\operatorname{dim}_{k} S_{i-1}\left(k^{n *}\right) \cdot \operatorname{dim}_{k}\left(\wedge^{n+i-1} k^{m}\right)=\left(\begin{array}{c}
n+i-2 \\
n-1
\end{array}\right) \cdot\left(\begin{array}{c}
m \\
n+i-1
\end{array}\right)
$$

Corollary 4.3. If $R$ is a GLCI ring of type $(m, n)$ with $n>1$, then

(1) $P_{R}(z)=(1+z)^{t} /\left(1-\sum_{i=1}^{m-n+1} c_{i} z^{i+1}\right)$ where $t=\operatorname{dim}_{k} \mathrm{~m} / \mathrm{m}^{2}=\operatorname{dim}_{k} \mathrm{~m}^{\prime} / \mathrm{m}^{\prime 2}$,

(2) $b_{i}=c_{1} b_{i-2}+c_{2} b_{i-3}+\cdots+c_{m-n+1} b_{i-m+n-2}+\left(\begin{array}{l}i \\ i\end{array}\right)$ where $b_{i}=b_{i}(R)$ is the ith Betti number of $R$,

(3) $\operatorname{Ext}_{R}(k, k)$ with the Yoneda product is finitely generated as an algebra and is generated by elements of degree $\leq t+1$.

Proof. By the results of Golod [8],

$$
P_{R}(z)=(1+z)^{t} /\left(1-\sum_{i=1}^{t} c_{i} z^{i+1}\right)
$$


if and only if $b_{i}=c_{1} b_{i-2}+c_{2} b_{i-3}+\cdots+c_{i} b_{i-1-1}+\left(\begin{array}{l}1 \\ i\end{array}\right)$ and each holds if and only if $H(K)$ has trivial Massey operations. These two formulas are therefore established by the first conclusion of the preceding theorem. Parts (1) and (2) of the corollary then follow from the fact that $c_{i}=0$ for $i>m-n+1$.

Part (3) also follows immediately from 4.2 and a theorem of Levin [10, Theorem 4.4.4] which states that if $R$ is a Golod ring, then (3) holds.

Corollary 4.4. Let $R$ be a regular local ring of dimension $t$ and let $a$ be an ideal generated by an $R$-sequence $a_{1}, \ldots, a_{d}$. Then $R / a^{n}$ is a GLCI ring of type $(d+n-1, n)$ and for $n>1, P_{R}(2)$ and $b_{i}(R)$ are given by (1) and (2) of Corollary 4.3 together with the formula for $c_{i}$ from Theorem 4.2 (with $d+n-1$ replacing $m$ in these formulas). In particular, $R / \mathfrak{m}^{n}$ is a GLCI ring of type $(t+n-1, n)$.

Proof. This corollary is really a special case of [5, Lemma 3.9] which says that $\mathrm{a}^{n}=I\left(\phi^{(n)}\right)$ where $\phi^{(n)}: R^{d+n-1} \rightarrow R^{n}$ is defined by the $(d+n-1) \times n$ matrix

$$
\left[\begin{array}{cccc}
a_{1} & 0 & \cdots & 0 \\
a_{2} & a_{1} & \cdots & 0 \\
\cdot & \cdot & & \cdot \\
\cdot & \cdot & & \cdot \\
\cdot & \cdot & & \cdot \\
a_{d} & a_{d-1} & \cdots & a_{d-n+1} \\
0 & a_{d} & \cdots & a_{d-n+2} \\
0 & 0 & \cdots & a_{d-n+3} \\
\cdot & \cdot & & \cdot \\
\cdot & \cdot & & \cdot \\
\cdot & \cdot & & \cdot \\
0 & 0 & \cdots & a_{d}
\end{array}\right]
$$

Equality of the ideals $a^{n}$ and $I\left(\phi^{(n)}\right)$ guarantees that $K\left(\wedge^{n} \phi^{(n)}\right)$ is acyclic which in turn says that $R / a^{n}$ is a GLCI ring of the desired type.

If $R=R^{\prime} / \mathrm{a}$ is a GLCI ring then a is a perfect ideal and so $R$ is CohenMacaulay. However, if $R$ is of type $(m, n)$ for $n>1$ (and so is a generalized local complete intersection but not a local complete intersection), then it is known that $R$ is not Gorenstein. Thus GLCI rings are not the much sought after intermediates between local complete intersections and Gorenstein rings. One indication of their bad behavior is given in the following example.

Example. We construct a ring of the form $R=R^{\prime} / \mathrm{a}$ which is not a GLCI ring but which nevertheless behaves like one with respect to the invariants discussed in this section. Consequently none of these invariants can by themselves characterize GLCI rings.

Let $R^{\prime}$ be a regular local ring of dimension $n$ and let $x_{1}, x_{2}$ be an $R^{\prime}$-sequence. Define the maps $f_{x}, f_{a x}: R^{\prime 3} \rightarrow R^{\prime 2}$ by the respective matrices 


$$
\left[\begin{array}{cc}
x_{1} & 0 \\
x_{2} & x_{1} \\
0 & x_{2}
\end{array}\right] \text { and }\left[\begin{array}{cc}
a x_{1} & 0 \\
a x_{2} & x_{1} \\
0 & x_{2}
\end{array}\right]
$$

where $a \in \mathfrak{m}^{\prime}$. Then $I_{x}=$ Ann Coker $\wedge^{2} f_{x}$ is of depth 2 while

$$
I_{a x}=\text { Ann Coker } \wedge^{2} f_{a x}=a \cdot I_{x}
$$

has depth 1 . Therefore $R^{\prime} / I_{x}$ is a GLCI ring of type $(3,2)$ while $R=R^{\prime} / I_{a x}$ is not a GLCI ring.

For the ring $R, H(K) \approx \operatorname{Tor}^{R^{\prime}}(R, k)$ can be computed using the following simple resolution: consider $K\left(\wedge^{2} f_{x}\right)$ which is a resolution of $R^{\prime} / I_{x}$ and, since $\operatorname{Ker} \wedge^{2} f_{x}=\operatorname{Ker} \wedge^{2} f_{a x}$, replace the map $\wedge^{2} f_{x}$ at the bottom of that complex by $\wedge^{2} f_{a x}$ to obtain the resolution $K_{R}$ of $R$. Since we now have $H(K) \approx$ $H\left(K_{R} \otimes_{R^{\prime}} k\right)$, it follows that $c_{i}(R)=c_{i}\left(R^{\prime} / I_{x}\right)$ for each $i$, and $c_{i}(R)=0$ for $i>2$.

We could now compute the multiplicative structure of $H(K)$ by thinking of $\left(K\left(\wedge^{2} f_{a x}\right), *\right)$ as a first approximation to an algebra resolution of $R$. Using the well-known method of Tate [12] to adjoin indeterminates $T_{i}$ (in this case of degree $\geq 2$ ) to kill all nonbounding cycles, we would obtain, as an algebra resolution of $R, \bar{K}_{R}=K\left(\wedge^{2} f_{a x}\right)\left\langle\cdots, T_{i}, \ldots\right\rangle$. Since $H(K) \approx H\left(\bar{K}_{R} \otimes_{R^{\prime}} k\right)$, it would follow that $H(K)$ has trivial multiplication and trivial higher order Massey operations making $R$ a Golod ring. This same result can be obtained immediately from a theorem of Shamash [10, Theorem 4.3.2] which says that for any $a \in \mathfrak{m}^{\prime}$ and any ideal $I \subset R^{\prime}, R^{\prime} / a \cdot I$ is a Golod ring.

The conclusions of 4.3 apply to all Golod rings and so $P_{R}(z), b_{i}(R)$, and $\operatorname{Ext}_{R}(k, k)$ all have the form given in Corollary 4.3 with $m=3$ and $n=2$. Moreover, $c_{i}(R)=c_{i}\left(R^{\prime} / I_{x}\right)$ for each $i$. Therefore none of the invariants considered in this section distinguish between $R^{\prime} / I_{x}$ which is a GLCI ring and $R=R^{\prime} / I_{a x}$ which is not.

\section{BIBLIOGRAPHY}

1. E. Assmus, On the homology of local rings, Illinois J. Math. 3 (1959), 187-199. MR 21 \#2670.

2. D. Buchsbaum, A generalized Koszul complex. I, Trans. Amer. Math. Soc. 111 (1964), 183-196. MR 28 \#3075.

3.—, Complexes associated with the minors of a matrix, Symposia Mathematica, vol. IV (INDAM, Rome, 1968/69), Academic Press, New York, 1970, pp. 255-283. MR 42 \# 7749.

4. D. Buchsbaum and D. Eisenbud, Remarks on ideals and resolutions, Symposia Mathematica (to appear).

5. D. Buchsbaum and D. Rim, A generalized Koszul complex. II. Depth and multiplicity, Trans. Amer. Math. Soc. 111 (1964), 197-224. MR 28 \#3076.

6.—, A generalized Koszul complex. III. A remark on generic acyclicity, Proc. Amer. Math. Soc. 16 (1965), 555-558. MR 31 \# 1285.

7. J. Eagon and D. Northcott, Ideals defined by matrices and a certain complex associated with them, Proc. Roy. Soc. London A 269 (1962), 188-204. MR 26 \# 161. 
8. E. Golod, On the homology of some local rings, Dokl. Akad. Nauk SSSR 144 (1962), 479-482 = Soviet Math. Dokl. 3 (1962), 745-748. MR 25 \#2110.

9. E. Gover, Generalized local complete intersections, Thesis, Brandeis University, Waltham, Mass., 1970.

10. T. Gulliksen and G. Levin, Homology of local rings, Queen's Paper, no. 20, Queen's University, Kingston, Ontario.

11. D. Quillen, On the (co-)homology of commutative rings, Proc. Sympos. Pure Math., vol. 17, Amer. Math. Soc., Providence, R.I., 1970, pp. 65-87. MR 41 \# 1722.

12. J. Tate, Homology of noetherian and local rings, Illinois J. Math. 1 (1957), 14-27. MR 19, 119.

Department of MATHEMATICS, Northeastern UNIVERstTy, Boston, MassachusetTs 02115 